\title{
Les connaissances acquises chez les lactocoques sont-elles transposables aux autres bactéries lactiques?
}

\author{
D Atlan \\ Laboratoire de microbiologie et génétique moléculaire (UMR CNRS 106), \\ Université Lyon I, 69622 Villeurbanne cedex, France
}

\begin{abstract}
Résumé - Les lactocoques sont les bactéries lactiques (BL) les mieux connues sur le plan fondamental. Afin de maîtriser les fermentations, il est essentiel de disposer d'une meilleure connaissance des souches bactériennes et de savoir si les connaissances acquises chez les lactocoques sont valables pour d'autres BL. Dans cette perspective, deux exemples sont choisis pour comparer plusieurs espèces de $\mathrm{BL}$ : des protéines situées à la surface cellulaire (protéines $S$ ou S-layer proteins, protéinases) ainsi que les systèmes de transport et de dégradation du lactose (système lac). Des différences peuvent exister en fonction du genre bactérien. Ainsi, les protéines $S$ identifiées chez de nombreuses bactéries recouvrent la surface des lactobacilles mais sont absentes chez les lactocoques. Une attention particulière est portée aux mécanismes d'attachement des protéines à la paroi bactérienne. Mais il existe aussi une grande variabilité au sein d'un même genre bactérien. Par exemple, deux systèmes lac sont principalement caractérisés chez les BL et présents chez le genre Lactobacillus. Les causes possibles de cette variabilité sont discutées.
\end{abstract}

\section{Lactococcus / Lactobacillus / protéinase / système lactose / protéines de surface}

Summary - Is the knowledge of lactococci sufficient to understand other lactic acid bacteria? Basically, lactococci are the best known lactic acid bacteria. A better control of fermentations involving these bacteria supposes to enlarge our knowledge of other lactic bacteria and to determine the properties they have in common. With this in view, two examples of comparison between lactococci and mainly lactobacilli are illustrated: cell surface-located proteins (S-layer proteins and proteinases) and lactose systems (transport and catabolism of lactose). Significant differences are noted between bacterial geni. S-layer proteins cover the cell surface of lactobacilli, but lactococci are devoid of this extra layer. Particular attention is given to mechanisms of protein binding to the cell wall. An intra-genus variability is also noted. For instance, two lactose sytems are mainly detected in lactobacilli. The origins of this variability are discussed.

Lactococcus / Lactobacillus / proteinase / lactose system / S-protein 


\section{INTRODUCTION}

Les bactéries lactiques (BL) sont caractérisées par la production d'acide lactique comme produit majeur de la fermentation des sucres. Ces bactéries anaérobies à Gram positif ont en commun des caractéristiques physiologiques et regroupent plusieurs genres bactériens : Lactococcus, Streptococcus, Lactobacillus, Pediococcus, Leuconostoc, Carnobacterium, Bifidobacterium, Enterococcus (Dellaglio et al, 1994). Une grande différence évolutive sépare ces bactéries dont le contenu GC s'étend de 33 à $67 \%$. Par exemple, Lactococcus lactis ssp cremoris (Lc cremoris) et Lactococcus lactis ssp lactis (LC lactis) sont caractérisées par un GC de 34 et $35 \%$, respectivement, alors que celui de lactobacillus ( $L b$ bulgaricus) est de $50 \%$ et que les bifidobactéries peuvent atteindre $67 \%$.

Sur le plan fondamental, le genre Lactococcus est fort étudié et fait figure de modèle ; plusieurs voies métaboliques et certains mécanismes ont été caractérisés : catabolisme du lactose, système protéolytique, biosynthèse des acides aminés branchés et des bactériocines, mécanismes de résistance aux phages. Depuis quelques années, l'étude de plusieurs genres bactériens s'est intensifiée, et tout particulièrement celle des lactobacilles. L'objectif de cette publication n'est pas de dresser une liste comparative des propriétés qui distinguent les lactocoques des autres BL. Deux exemples ont été choisis et concernent les protéines localisées à la surface bactérienne et le système de transport et de dégradation du lactose. Une large part est donnée au genre Lactobacillus.

\section{COMPARTIMENTATION ET ANCRAGE DE PROTÉINES SITUÉES À LA SURFACE BACTÉRIENNE}

L'enveloppe des bactéries à Gram positif est constituée d'une membrane cytoplas- mique et de la paroi. La membrane cytoplasmique est composée d'une double couche de phospholipides qui forme une barrière sélective et renferme de nombreuses protéines qui interviennent dans différents processsus biologiques essentiels (production d'énergie...). D'une épaisseur de 20 à $40 \mathrm{~nm}$, la paroi est responsable de la forme et de la rigidité bactériennes. Elle est constituée du peptidoglycane qui forme un réseau tridimensionnel abritant des protéines, des acides téichoïques et lipotéichoïques (Shockman et Barrett, 1983). Le peptidoglycane, ou muréine, est un hétéropolymère dont les chaînes principales sont constituées de $\mathrm{N}$ acétylglucosamine et d'acide $\mathrm{N}$-acétylmuramique et les chaînes latérales de peptides. Seule la composition des chaînes peptidiques varie et constitue un critère taxonomique permettant de distinguer deux classes de BL selon la présence d'acide mésodiaminopimélique ou de lysine.

La surface d'un grand nombre de bactéries (archae- et eubactéries) est recouverte d'une couche protéique organisée en un réseau régulier, correspondant à des répétitions d'unités protéiques (ou glycoprotéiques), appelées protéines de surface ou protéines S (S-layer proteins dans la littérature) (Sleytr et Messner, 1988). Ces protéines $\mathrm{S}$ ont la propriété de s'assembler in vivo et in vitro selon une symétrie qui varie avec l'espèce bactérienne. Elles sont liées entre elles et à d'autres composants de l'enveloppe par des liaisons non covalentes. Ces protéines multifonctionnelles sont impliquées dans le criblage des solutés externes (seuil d'exclusion de 40 à $80000 \mathrm{Da}$ ), des processus d'échanges d'ions, d'interaction avec les bactériophages, d'adhésion cellulaire et de pathogénicité.

Les protéines situées à la surface bactérienne sont synthétisées dans le cytoplasme et le plus souvent caractérisées par des signaux qui sont nécessaires à l'expor- 
tation et la compartimentation dans l'enveloppe bactérienne.

\section{Les différents signaux caractéristiques des protéines de surface}

\section{Signal d'exportation}

Chez les bactéries à Gram positif ou négatif, un grand nombre de protéines sont exportées grâce au système général de sécrétion Sec (Pugsley, 1993 ; Simonen et Palva, 1993). L'extrémité $\mathrm{NH}_{2}$ des protéines exportées présente une séquence caractéristique, nommée séquence signal (région "pré") qui est éliminée après transfert à travers la membrane cytoplasmique. On pense que la séquence signal ralentirait le repliement des polypeptides nouvellement synthétisés permettant ainsi leur reconnaissance par une protéine cytoplasmique chaperon (SecB) et leur maintien dans une configuration compatible avec la traversée de la membrane. La longueur des séquences signal varie de 18 à 35 acides aminés et on distingue trois domaines caractéristiques. La région $\mathrm{N}$-terminale est courte, hydrophile et contient au moins un résidu chargé positivement. Le second domaine (12 à 15 résidus) est le plus long, hydrophobe avec une configuration en hélice $\alpha$. Enfin, un court domaine (quatre à six résidus) contient une séquence consensus de type Ala-X-Ala reconnue par les peptidases LepB ou LspA.

\section{Signaux de compartimentation}

\section{Motif LPXTG}

Outre la fonction de code postal assurée par la séquence signal, des études récentes ont permis de mettre en évidence chez de nombreuses protéines de bactéries à Gram positif la présence d'un signal de compartimentation et d'ancrage dans la paroi bac- térienne (Fischetti et al, 1991 ; Schneewind et al, 1993). On peut citer parmi les plus étudiées la protéine A de Staphylococcus aureus, la protéine M6 de Streptococcus pyogenes et la protéine $\mathrm{G}$ des streptocoques du groupe $\mathrm{G}$. Leur partie C-terminale présente une forte homologie et peut être divisée en trois régions. La première est de longueur variable, riche en proline, thréonine, glycine et sérine et contient un seul motif très conservé : Leu-Pro-X-ThrGly (LPXTG). Neuf résidus en aval de ce motif, on trouve une région très hydrophobe de 18 à 22 résidus qui forme une hélice $\alpha$. Enfin, l'extrémité $\mathrm{COOH}$ (cinq à sept résidus) est chargée et constitue une séquence "stop transfert".

Navarre et Schneewind (1994) ont montré que le motif LPXTG constitue un signal spécifique de reconnaissance et de compartimentation dans la paroi bactérienne. En effet, l'entérotoxine B, protéine normalement excrétée dans le milieu, reste localisée dans la paroi lorsque le signal de compartimentation est ajouté à sa séquence. Un modèle rendant compte d'un mécanisme général de compartimentation et d'ancrage dans la paroi bactérienne a été proposé pour les protéines portant le motif LPXTG. Lorsqu'un polypeptide traverse la membrane cytoplasmique, le motif LPXTG serait reconnu par un complexe enzymatique ("Sortase") situé sur la face externe de cette membrane. Les résidus basiques de la région carboxy-terminale stopperaient la progression de la chaîne polypeptidique par interaction avec les phosphates de la bicouche de phospholipides. Puis la sortase couperait le polypeptide entre la thréonine et la glycine du motif LPXTG. La thréonine se lierait par son extrémité $\mathrm{COOH}$ libre au groupement amine de l'un des acides aminés des ponts inter-peptidiques du peptidoglycane. Cette liaison covalente empêcherait la libération des protéines dans le milieu extracellulaire. 


\section{Domaines SLH}

Les domaines SLH (S-layer homology), ne présentent pas de séquence consensus mais une similitude avec la partie $\mathrm{N}$-terminale de certaines protéines de surface (Acetogenium kiwi, Bacillus sphaericus, Thermus thermophilus et Bacillus brevis). Le modèle du cellulosome de Clostridium thermocellum permet de comprendre la fonction des domaines SLH (Fujino et al, 1993; Lemaire et al, 1995), Ce cellulosome est un complexe cellulolytique situé en protubérance à la surface cellulaire et ensuite libéré dans le milieu de culture. Les différents constituants du cellulosome sont sécrétés individuellement, puis s'assemblent à la surface cellulaire autour de la protéine CipA. La protéine OlpA, est une protéine de surface dont l'extrémité $\mathrm{NH}_{2}$ sert de récepteur à la protéine CipA, grâce à un segment de 22 résidus (retrouvés en plusieurs exemplaires chez CipA). La partie C-terminale d'OlpA comprend un domaine riche en proline, glycine, thréonine et sérine, suivi de trois séquences répétées de 65 résidus correspondant à des domaines SLH. Il a été prouvé que ces domaines SLH sont responsables de l'attachement à l'enveloppe par des liaisons non covalentes avec le peptidoglycane ; ainsi une protéine chimérique dont la partie $\mathrm{C}$-terminale de MalE (protéine fixatrice du maltose) a été remplacée par un domaine SLH de la protéine OlpB du cellulosome est bien détectée à la surface cellulaire. Chez B sphaericus, la protéine S délétée de la partie $\mathrm{N}$-terminale comprenant le domaine SLH est capable de constituer un réseau cristallin mais n'est plus liée au peptidoglycane (Hastie et Brinton, 1979).

Les domaines SLH sont présents chez plusieurs enzymes extracellulaires agissant sur des polymères (xylanase, cellulase, pullulanase). Ils sont supposés servir de signal de sortie pour les protéines exocellulaires comme le fait le motif LPxTG. II est probable que la liaison avec le peptidoglycane ne soit que transitoire.

\section{Liaison à l'acide lipotéichoïque (ALT)}

Ce type de liaison requiert la présence de choline dans les acides téichoïques ce qui est assez rare chez les bactéries à Gram positif et caractéristique des streptocoques. Le mécanisme a été démontré grâce à l'étude de la protéine de surface PspA de $S$ pneunomiae qui est aussi impliquée dans la virulence de cette bactérie (Yother et White, 1994). PspA possède une séquence signal $\mathrm{N}$-terminale et sa région $\mathrm{C}$-terminale contient un domaine riche en proline constitué d'une part de dix unités très conservées de 20 acides aminés et d'autre part d'un domaine très court, peu chargé et légèrement hydrophobe. PspA peut-être complètement excrétée dans trois conditions : délétion des 5 dernières unités répétées, absence totale de choline dans le milieu de culture ou présence d'une forte concentration $(>0,5 \%)$. Seules les 5 dernières unités de PspA sont nécessaires à l'attachement au ALT par l'intermédiaire de la choline de ce composé. Sur le chromosome de $S$ pneumoniae, il y a plusieurs séquences homologues à celles de PspA.

\section{Protéinases des lactocoques et des lactobacilles}

Le développement des lactocoques et des lactobacilles dans le lait est possible grâce à un système protéolytique complexe qui assure les besoins en acides aminés essentiels à partir de la dégradation des caséines. La première étape est réalisée par une endoprotéase, nommée protéinase (PrtP), située à la surface bactérienne et indispensable pour la croissance dans le lait. Chez les lactocoques, diverses approches (biochimique, génétique, moléculaire et immunologique) ont permis de bien caractériser cette enzyme. Les séquences des gènes de structure sont disponibles pour trois lactocoques et montrent que les PrtPs sont synthétisées sous forme de préproprotéines 
d'environ 210 kDa ( Kok et al, 1988 ; Kiwaki et al, 1989 ; Vos et al, 1989). Les séquences peptidiques des PrtPs sont très conservées : on note seulement 18 résidus différents entre $L c$ cremoris $\mathrm{Wg} 2$ et $L c$ lactis NCDO763. Les PrtPs diffèrent par leur spécificité d'hydrolyse des caséines, et récemment plusieurs domaines protéiques responsables de cette propriété ont été identifiés (Siezen et al, 1993 ; Bruinenberg et al, 1994).

Les PrtPs possèdent une séquence signal $\mathrm{N}$-terminale de 33 résidus, On distingue aussi à l'extrémité $\mathrm{COOH}$ le motif spécifique LPXTG ainsi que la séquence complète de compartimentation et d'ancrage associée à ce motif. On peut donc penser que selon le modèle de Navarre et Schneewind (1994), les protéinases des lactocoques sont associées de façon covalente au peptidoglycane. Néanmoins, dans certaines conditions (absence d'ions $\mathrm{Ca}^{++}$), les PrtPs peuvent être libérées dans le mileu extracellulaire par auto-protéolyse de la partie carboxy-terminale, ce qui conduit à une enzyme de $145 \mathrm{kDa}$ toujours active, voire de 80-82 kDa.

Les propriétés biochimiques de la protéinase de $L b$ paracasei et la séquence de l'enzyme révèlent une forte identité avec les PrtPs de lactocoques (Holck et Naes, 1992). Ces données suggèrent que la première étape du catabolisme des caséines est identique chez les lactocoques et les lactobacilles. L'étude récente de protéinases de lactobacilles thermophiles a permis de mettre en évidence des différences significatives au niveau de la spécificité d'hydrolyse, l'autoprotéolyse et de l'attachement à l'enveloppe bactérienne.

Ainsi, la spécificité d'hydrolyse de la protéinase de $\angle b$ helveticus CNRZ303 sur le fragment 1-23 de la caséine $\alpha_{s 1}$ est très restreinte et, de plus, elle présente un site de coupure original jamais observé chez les PrtPs (Zevaco et Gripon, 1988). Les protéinases de $L b$ helveticus $L 89$ et $L b$ bulga- ricus CNRZ397 sont incapables de se libérer de la paroi bactérienne par autoprotéolyse de leur extrémité $\mathrm{COOH}$ (Martin-Hernandez et al, 1994 ; Laloi et al, 1991). L'extraction de la protéinase de la paroi de Lb bulgaricus nécessite un traitement des cellules avec une muramidase (lysozyme) puis un choc osmotique. Cette protéinase présente aussi la particularité d'être sensible aux inhibiteurs des protéases à cystéine et à sérine. Récemment, l'analyse de la séquence peptidique a montré une homologie significative de la partie $\mathrm{N}$-terminale et du domaine catalytique avec les PrtPs des lactocoques (Gilbert et al, résultats non publiés). Au contraire, l'extrémité $\mathrm{COOH}$ diffère de celle des PrtPs : seul un motif d'ancrage dégénéré est présent entre deux régions répétées de 41 résidus, avec une forte proportion de lysine. On peut donc penser que le mécanisme d'attachement de la protéinase de $L b$ bulgaricus est différent de celui des PrtPs des autres BL. De plus, il semblerait que Lb bulgaricus possède dans sa paroi une autre endoprotéase de type metalloprotéase, dont on ne connaît pas l'implication dans la voie caséinolytique (Stefanisi et al, 1995).

Les protéinases des lactocoques et lactobacilles appartiennent à la famille des subtilisines de par leur homologie avec le site actif impliquant trois acides aminés (sérine, acide aspartique et histidine). Elles se distinguent des autres enzymes de cette famille selon deux critères principaux :

- synthèse pendant la phase exponentielle de croissance ;

- présence d'une longue extension C-terminale et attachement à l'enveloppe bactérienne.

Deux enzymes de cette famille, à savoir une protéase à sérine mineure (Epr) et la bacillopeptidase $F$ possèdent une partie $C$ terminale plus longue que les subtilisines sensu stricto, et sont douées comme les PrtPs de la propriété d'auto-digestion de l'extrémité $\mathrm{COOH}$ (He et al, 1991). À cet 
égard, la protéinase de $L b$ bulgaricus est donc une exception à la famille des subtilisines.

Le système protéolytique des lactobacilles thermophiles se distingue aussi de celui des lactocoques par la présence de plusieurs peptidases dans l'enveloppe bactérienne : aminopeptidases générales et proline iminopeptidase (Atlan et al, 1989 ; Blanc et al, 1993 ; Atlan et al, 1994).

\section{Protéines S des lactobacilles}

De très nombreuses espèces du genre Lactobacillus ( $L b$ brevis, fermentum, helveticus, acidophilus, buchneri) possèdent une couche externe de 5 à $15 \mathrm{~nm}$ d'épaisseur, organisée en un réseau régulier avec une symétrie oblique (type p2), carrée (p4) ou hexagonale (p6) (Lortal, 1993). Cette couche protéique est constituée d'une seule protéine et peut être dissociée de la paroi par un traitement avec des agents capables de rompre des liaisons hydrogènes (urée, hydrochlorure de guanidine) ou des détergents (SDS). D'une masse de 40 à $60 \mathrm{kDa}$, les protéines S représentent 7 à $14 \%$ du contenu protéique total de la bactérie. Elles renferment de nombreux acides aminés hydrophobes (valine et alanine) et basiques (lysine). Les gènes codant pour les protéines $\mathrm{S}$ de $L b$ brevis, acidophilus et helveticus ont été récemment séquencés (Vidgren et al, 1992 ; Boot et al, 1993). L'extrémité $\mathrm{NH}_{2}$ des protéines de surface présente une séquence signal caractéristique. Une forte homologie est observée entre les séquences peptidiques des protéines $\mathrm{S}$ des lactobacilles thermophiles (acidophilus et helveticus). La protéine S de $L b$ acidophilus présente une identité significative avec celles de $L b$ brevis et $B$ sphaericus (36 et $42,5 \%$, respectivement) ; elle est caractérisée par la présence de domaines SLH décrits chez plusieurs protéines de surface (voir paragraphe Domaines SLH) qui pourraient donc être responsables de l'attachement à l'enveloppe bactérienne.

L'organisation de la surface bactérienne s'avère différente chez les lactocoques et les lactobacilles. L'étude des protéines $\mathrm{S}$ et des protéinases montrent que les mécanismes d'attachement varient selon le type de la protéine, mais aussi pour une protéine donnée selon l'espèce bactérienne.

\section{SYSTĖME LACTOSE : TRANSPORT ET CATABOLISME}

Une des fonctions primaires des BL lors de la fermentation du lait est la dégradation du lactose en acide lactique et parfois autres produits. II existe principalement deux types de systèmes de transport et catabolisme chez les BL. Les gènes impliqués dans le système lac ont été identifiés et séquencés chez plusieurs espèces, et sont souvent associés aux gènes codant pour la dégradation du galactose (revue : de Vos et Vaughan, 1994).

\section{Système PTS et phospho- $\beta$-galactosidase}

Le système de phosphotransférase (PTS) est spécifique du lactose, dépendant du phosphoénolpyruvate et conduit à la phosphorylation du lactose en lactose-6-phosphate. Ce système est constitué de deux protéines générales cytoplasmiques (Enzyme let HPr) et de deux composantes spécifiques du lactose, la protéine membranaire LacE et le phosphotransporteur LacF (appelés précédemment Enzyme Illac et IIIlac, respectivement). Le lactose-6P est ensuite hydrolysé par une phospho- $\beta$-galactosidase ( $P$ - $\beta$ Gal) (LacG) générant du galactose- $6 \mathrm{P}$, ce dernier suivant la voie du tagatose-6P. Cette voie métabolique comprend 3 enzymes : galactose- $6 \mathrm{P}$ isomérase 
(LacAB), tagatose-6P kinase ( $\mathrm{LacC}$ ) et tagatose-1,6-diP aldolase (LacD).

Ce système spécifique de transport est exclusivement présent chez les bactéries à Gram positif. L'organisation et le contrôle de l'opéron lac de Lc lactis sont proches de ceux de Staphylococcus aureus et Streptococcus mutans. En effet, les gènes codant pour les composantes spécifiques du système PTS, la P- $\beta$ Gal et les enzymes de la voie du tagatose-6P font partie du même opéron et il existe une forte homologie au niveau des enzymes (jusqu'à $84 \%$ ). Chez Lc lactis, cet opéron est exprimé lorsque les bactéries se développent en présence de lactose, et il est sous le contrôle d'un répresseur, produit du gène lach, situé immédiatement en amont et en sens inverse de l'opéron lac; néanmoins, l'inducteur vrai est le tagatose-6P. Si, comme chez Lc lactis, l'opéron lac de $\mathrm{Lb}$ casei $64 \mathrm{H}$ est porté par un plasmide, son organisation est différente. La localisation des gènes responsables de la voie du tagatose-6P est encore inconnue et l'inducteur n'est pas le tagatose- $6 \mathrm{P}$; le produit du premier gène de l'opéron lac de Lb casei (lacT), contrôle probablement l'expression des gènes lac. La similitude avec les enzymes du système lac de Lc lactis varie de 43 à $54 \%$.

\section{Perméase LacS et $\beta$-galactosidase}

Le lactose n'est pas modifié lors de son transport par la perméase LacS, localisée dans la membrane cytoplasmique. Cette perméase fonctionne comme un système de symport lié à l'entrée simultanée d'ions $\mathrm{H}^{+}$; dans le cas d'une forte accumulation intracellulaire de galactose, elle agit comme un antiport lié à la sortie de galactose. Cette protéine de 634 résidus comprend un domaine hydrophobe $\mathrm{N}$-terminal qui assume la fonction de transporteur et ressemble à la perméase du mélibiose chez E coli ; la partie C-terminale de LacS est hydrophile et caractérisée par une homologie avec la phosphotransférase LacE.

Chez $S$ thermophilus et $L b$ bulgaricus, le lactose entre grâce à la perméase LacS, puis est dégradé par une $\beta$-galactosidase ( $\beta$-Gal) codée par le gène lac $Z$. Le galactose obtenu n'est pas utilisé (phénotype $\mathrm{Gal}^{-}$de ces deux espèces), mais excrété par la perméase LacS. Cette perméase n'est pas spécifique du transport du lactose ; en effet, il a été prouvé chez $S$ thermophilus que LacS présente une forte affinité pour d'autres $\beta$-galactosides (mélibiose, galactose, raffinose). Chez ces deux espèces thermophiles, les gènes lac $S$ et lac $Z$ forment un opéron analogue et la transcription démarre à partir d'un promoteur situé juste en amont de lacS. Chez $L b$ bulgaricus, l'expression des gènes lac est constitutive, tandis que celle de $S$ thermophilus est induite par le galactose et ses analogues plutôt que le lactose et réprimée par le glucose. En amont de l'opéron lac de $S$ thermophilus, sont présents certains gènes de la voie de dégradation du galactose (ga/T, galE, galM) (voie de Leloir). II manque le gène galK ce qui pourrait être une explication au phénotype $\mathrm{Gal}^{-}$.

Les deux protéines (LacS et $\beta$-Gal) du système lac de $L b$ bulgaricus et $S$ thermophilus sont très homologues (monomère de 1006 et 1023 acides aminés, respectivement). LacS ne présente aucune homologie avec la perméase LacY d'E coli. La $\beta$ Gal de $L b$ bulgaricus ne présente qu'une similitude de $30 \%$ avec l'enzyme d'E coli.

Cette organisation de l'opéron lac et le phénotype $\mathrm{Gal}^{-}$ne sont pas une règle générale des $B L$ thermophiles. En effet, $L b$ helveticus est capable de dégrader le galactose et possède tous les gènes nécessaires à la voie de Leloir, mais l'organisation du système lac est différente de celle de $S$ thermophilus. D'une part, le gène galK est présent et l'ordre des gènes gal est différent. D'autre part, la $\beta$-Gal est constituée de deux sous-unités, LacL et LacM, codées par des 
gènes se recouvrant partiellement. Ce type de $\beta$-Gal se retrouve chez d'autres $B L: L b$. plantarum, Lb casei ATCC313, Leuconostoc lactis. Les séquences des protéines LacL et $M$ possèdent de fortes homologies avec les $\beta$-Gals de type LacZ de $S$ thermophilus, Lb bulgaricus et Clostridium acetobutylicum: en particulier les régions $\mathrm{N}$ - et $\mathrm{C}$-terminales et les résidus du site actif (Glu461 et Tyr503 d'E coli). La taille de LacL et M est plus faible que celle du produit du gène lac $Z$.

L'ensemble des données présentées montre que les composantes du système lac sont différentes au sein des BL, mais aussi d'un genre bactérien voire d'une espèce. Parfois plusieurs systèmes lac peuvent coexister au sein d'une même souche. Ainsi, Lc lactis ATCC 7962 (non laitière) possède d'une part le système PTS avec une très faible activité $P$ - $\beta$ Gal, et d'autre part une perméase, LacY, fonctionnant comme un système de symport lié à l'entrée de protons et une $\beta-G a l$. Certaines souches de Lc lactis posséderaient une perméase codée par un gène chromosomique.

Étant donné la conservation des gènes lac, on peut avancer l'hypothèse d'un transfert horizontal de gènes entre les BL mésophiles ( $L c$ lactis et Lb paracasei) et d'autres bactéries à Gram positif (Staphylococcus aureus et Streptococcus mutans), transfert dans lequel les BL thermophiles ( $S$ thermophilus, Lb bulgaricus, $L b$ helveticus) n'auraient pas été impliquées. Ce transfert serait récent et favorisé par la localisation plasmidique des gènes ou une proximité d'éléments mobiles de type IS. Cette hypothèse est aussi renforcée par l'analyse des protéinases. Le gène prtP est le plus souvent porté par le mème plasmide que le système lac chez les BL mésophiles citées précédemment. Chez la sous-espèce thermophile bulgaricus, le gène codant pour la protéinase, différente des PrtPs, est localisé sur le chromosome en aval de l'opéron lac et séparé par un élément IS spécifique à bulgaricus, ISL3 (Germond et al, 1995). Lb bulgaricus se différencie donc totalement des lactocoques et lactobacilles mésophiles pour les deux systèmes essentiels au développement bactérien dans le lait, à savoir les sytèmes lac et prt.

L'étude de différentes souches n'appartenant pas au genre Lactococcus met en évidence une variabilité d'adaptation des bactéries lactiques à un nouvel environnement, le lait, et à son utilisation par l'acquisition de gènes provenant d'espèces du même biotope. Cette variété constitue un atout majeur pour l'amélioration biotechnologique des souches par modification de leur patrimoine génétique.

\section{RÉFÉRENCES}

Atlan D, Gilbert C, Blanc B, Portalier R (1994) Cloning, sequencing and characterization of a proline iminopeptidase from Lactobacillus delbrueckii subsp bulgaricus CNRZ397. Microbiology 140, 537-542

Atlan D, Laloi P, Portalier R (1989) Isolation and characterization of aminopeptidase-deficient Lactobacillus buigaricus mutants. Appl Environ Microbiol 55, $1717-1723$

Blanc B, Laloi P, Atlan D, Gilbert C, Portalier A (1993) Two cell wall-associated aminopeptidases from Lactobacillus helveticus and the purification and characterization of APII from strain ITGL1, J Gen Microbiol 139, 1441-1448

Boot H, Kolen C, van Noort J, Pouwels P (1993) S-layer protein of Lactobacillus acidophilus ATCC4356: purification, expression in Escherichia coli, and nucleotide sequence of the corresponding gene. J Bacteriol $175,6089-6096$

Bruinenberg P, Doesburg P, Alting A, Exterkate F, de Vos W, Siezen, R (1994) Evidence for a large dispensable segment in the subtilisin-like catalytic domain of the Lactococcus lactis cell-envelope proteinase. Protein Eng 7, 991-996

Dellaglio F, de Roissart H, Torriani S, Curk M, Janssens D (1994) Caractéristiques générales des bactéries lactiques. In : Bactéries Lactiques (Luquet FM, de Roissart $\mathrm{H}$, eds) Lorica, Uriage

de Vos W, Vaughan E (1994) Genetics of lactose utilization in lactic acid bacteria. FEMS Microbiol Rev $15,217-237$.

Fischetti V, Pancholi V, Schneewind O (1991) Common characteristics of the surface proteins from Grampositive cocci. In: Genetics and molecular biology of streptococci, lactococci and enterococci (Dunny 
C, Mc Kay L, eds) American Society for Microbiology, Washington DC

Fujino T, Béguin P, Aubert JP (1993) Organization of a Clostridium thermocellum gene cluster encoding the cellulosomal scaffolding protein $\mathrm{CipA}$ and a protein possibly involved in attachment of the cellulosome to the cell surface. J Bacteriol 175, 1891-1899

Germond JE, Lapierre L, Delley M, Mollet B (1995) A new mobile element in Lactobacillus delbrueckii subsp bulgaricus. Mol Gen Genet 248, 407-416

Hastie AT, Brinton Jr CC (1979) Specific interaction of the tetragonally arrayed protein layer of Bacillus sphaericus with its peptidoglycan sacculus. $J$ Bacteriol $138,1010-1021$

He XS, Brückner R, Doi R (1991) The protease genes of Bacillus subtilis. Res Microbiol 142, 797-803

Holck A, Næs H (1992) Cloning, sequencing and expression of the gene encoding the cell-envelope-associated proteinase from Lactobacillus paracasei subsp paracasei NCDO151. J Gen Microbiol 138, 13531364

Kiwaki M, Ikemura H, Shimizu-Kadota M, Hirashima A (1989) Molecular characterization of a cell-wall associated proteinase gene from Streptococcus lactis NCDO763. Mol Microbiol 3, 359-369

Kok J, Leenhouts K, Haandrikman A, Ledeboer A, Venema G (1988) Nucleotide sequence of the cellwall proteinase gene of Streptococcus cremoris Wg2. Appl Environ Microbiol 54, 231-238

Laloi P, Atlan D, Blanc B, Gilbert C, Portalier R (1991) Cell-wall associated proteinase of Lactobacillus delbrueckii subsp bulgaricus CNRZ397: differential extraction, purification and properties of the enzyme. Appl Microbiol Biotechnol 36, 196-204

Lemaire $M$, Ottayon $H$, Gounon P, Fujino T, Béguin P (1995) OlpB, a new outer layer protein of Clostridium thermocellum, and binding of its S-layer like domains to components of the cell enveloppe. J Bacteriol 177, 2451-2459

Lortal S (1993) Crystalline surface-layers of the genus Lactobacillus. In: Advances in bacterial paracrystalline surface layers (Beveridge TJ, Kloval SF, eds). Plenum Press, New York

Martin-Hernandez C, Alting A, Exterkate F (1994) Purification and characterization of the mature, mem- brane associated cell-envelope proteinase of Lactobacillus helveticus L89. Appl Microbiol Biotechnol $40,828-834$

Navarre W, Schneewind O (1994) Proteolytic cleavage and cell wall anchoring at the LPXTG motif of surface proteins in gram-positive bacteria. Mol Microbiol 14, 115-121

Pugsley A (1993) The complete general secretory pathway in Gram-negative bacteria. Microbiol Rev 57 , 50-108

Schneewind O, Mihaylova-Petkov D, Model P (1993) Cell wall sorting signals in surface proteins of Grampositive bacteria. EMBO J 12, 4803-4811

Shockman G, Barrett J (1983) Structure, function and assembly of cell walls of Gram-positive bacteria. Annu Rev Microbio/ 37, 501-527

Siezen R, Bruinenberg P, Vos P, van Alen-Boerrigter I. Nijhuis M, Alting A, Exterkate F, de Vos W (1993) Engineering of the substrate-binding region of the subtilisin-like, cell-envelope proteinase of Lactococcus lactis. Protein Eng 6, 927-937

Simonen M, Palva I (1993) Protein secretion in BacilIus species. Microbiol Rev 57, 109-137

Sleytr U, Messner $P$ (1988) Crystalline surface layers in procaryotes. J Bacteriol 170, 2891-2897

Stefanisi D, Sakellaris G, Garel JR (1995) The presence of two proteinases associated with the cell wall of Lactobacillus bulgaricus. FEMS Microbiol Lett 128 , 53-58

Vidgrén G, Palva I, Pakkanen R, Lounatmaa K, Palva A (1992) S-layer gene of Lactobacillus brevis: Cloning by polymerase chain reaction and determination of the nucleotide sequence. J Bacteriol 174 , 7419-7427

Vos P, Simons G, Siezen R, deVos W (1989) Primary structure and organization of the gene for procaryotic cell envelope-located serine proteinase. $J$ Biol Chem 264, 13579-13585

Yother J, White J (1994) Novel attachment mechanism of the Streptococcus pneunomiae protein PspA. J Bacteriol 176, 2976-2985

Zevaco C, Gripon JC (1988) Properties and specificity of a cell-wall proteinase from Lactobacillus helveticus. Lait 68, 393-408 